\title{
An infant with tachypnoea
}

\section{Presenting complaint}

A 4-month-old young male was referred to the paediatric outpatient clinic of allergology and pulmonology due to "abdominal breathing".

\section{Past medical history}

The patient was delivered by elective Caesarean section at 34 week's gestation due to newly diagnosed maternal Hodgkin's disease. The Apgar score was 6, 9 and 10 at 1, 5 and 10 minutes, respectively. A chest radiograph on day 1 demonstrated a typical pattern of respiratory distress syndrome (RDS), and he received ventilator/continuous positive airway pressure support for 48 hours and extra oxygen was required for the first 14 days of life. The RDS had improved, but chest radiography was not completely normal, 2 weeks later. The patient was discharged at 3 weeks of age with, as the mother reported, "a little rapid breathing, but otherwise completely normal".

\section{History after neonatal period}

The patient was referred to the outpatient clinic at 4 months of age due to "abdominal breathing". The mother reported somewhat "rapid breathing", and some or slight abdominal breathing since discharge from hospital. Apart from that the parents considered him to be entirely well. The parents denied any further respiratory symptoms, in particular no cough, respiratory discomfort, airways secretions, cyanosis or sweating. He gained weight and was a happy, smiling baby. He had no skin disease, no gastrointestinal or urinary tract system symptoms and no renal disease, but he was, of course, not breastfed because of maternal treatment.

\section{Family history}

The patient was the first child of native, Norwegian parents with no lung diseases or atopic diseases in the family. However, a few weeks after the first contact, in the pollen season, the mother developed symptoms of allergic rhinoconjunctivitis for the first time. The mother's Hodgkin's disease was diagnosed in January 2004, although symptoms had been present from November 2003. There were no pets in the family home and no one smoked.

\section{Clinical findings}

On clinical examination, the patient presented as well nourished and happy, with normal weight and length for his age, normal skin and skin colour. Respiratory rate was $50-60$ breaths per minute, increasing to 60 breaths per minute with slight activity. He had some sub-costal, but no intra-costal recessions. Auscultatory findings included prolonged expiration, normal vesicular respiratory sounds and no additional breath sounds (including no crepitations). Heart sounds were normal, whereas the liver was palpable $\sim 1.5-2 \mathrm{~cm}$ under the costal border. However, with no abdominal mass or organomegaly this was believed to be caused by hyperinflation and subsequent liver displacement. Neurological examination was normal.

Thus, at 4 months, the patient presented with tachypnoea and slight pulmonary hyperinflation.

\section{Task 1}

At this stage, what would you do?

1. Wait and observe.

2. Perform a blood test.

3. Do a chest radiograph.

4. Do a cardiac investigation.

5. Measure arterial oxygen saturation.

\section{K.C. Lødrup Carlsen}

Section of Allergology and Pulmonology

Dept of Paediatrics

Ullevål University Hospital

NO-0407 Oslo, Norway

Fax: 4722118663

Email:kclo@uus.no
This case presentation was taken from a Ground Round, held at the ERS Congress, Glasgow 2004. Go to www.ersnet.org /elearning for further information. 
Table 1

Differential diagnoses of interstitial lung disease in children

\begin{tabular}{l} 
Paediatric-acquired \\
immunodeficiency syndrome \\
Cystic fibrosis \\
Gastro-oesophageal \\
reflux/chronic aspiration \\
Immunodeficiency syndromes \\
Ciliary disorders \\
Collagen vascular disorders \\
Hypersensitivity pneumontis \\
Pulmonary haemosiderosis \\
Post-infectious pneumonitis \\
\hline Reproduced with permission from \\
Pediatric Review [1].
\end{tabular}
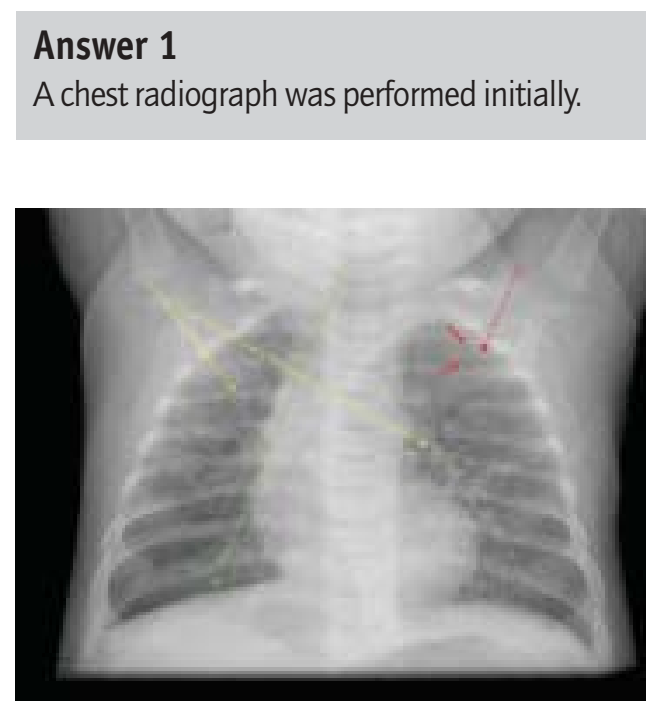

Figure 1

Chest radiograph at 4 months.

The chest radiograph was somewhat surprising, with suggestions of diffuse interstitial infiltrates (arrow from right side of chest), hyperinflation (middle arrow) and there appeared to be emphysematous bullae (three arrows).

\section{Differential diagnosis}

At this stage, the differential diagnoses considered were: chronic lung disease of the newborn, masqueraders of interstitial lung disease in infants, (chronic) interstitial lung disease or interstitial lung disease associated with systemic disease. Some further possibilities are presented in table 1.

\section{Additional investigations}

Subsequently, the patient underwent cardiac investigation, ruling out underlying heart disease. Oxygen saturation measurements over 3 days were normal, with a few short dips $<90$ only when he was very active. Both skin-prick tests and specific IgE were completely negative for common allergens, including moulds, with the consideration of allergic alveolitis or any other allergic sensitisation pneumonias. Sweat test results were normal. Tidal flow-volume (TFV) loops indicated bronchial obstruction (figure 2).

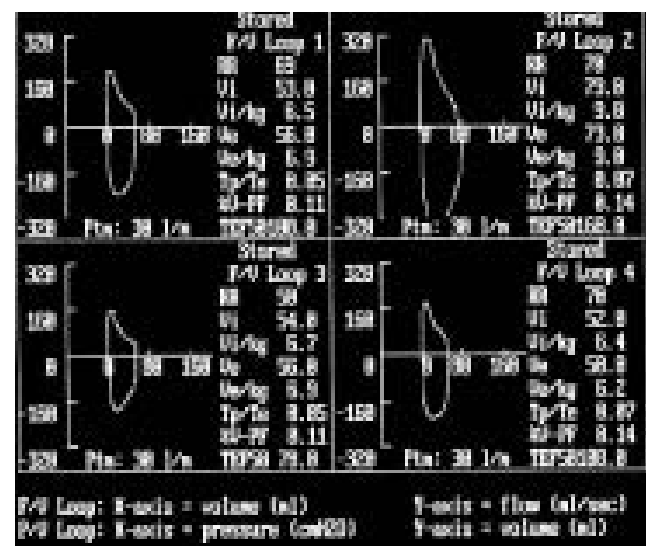

Figure 2

Tidal flow volume loops (measured awake).

Time to peak expiratory flow/total expiratory time ( $t$ PTEF/ $t E$ ) was reduced and the TFV curve was concave, indicating bronchial obstruction.

\section{Blood tests}

The patient's blood tests were normal in most respects. They showed normal haematology, including differential cell counts, immunoglobulins $E, A, M$ and $G$, including sub-classes, and $\alpha_{1}$-antitrypsin. He had a negative antinuclear antibodies, and negative serology for Epstein Barr virus (EBV), toxoplasmosis, respiratory synctial virus, adenovirus, parainfluenza virus, HIV, mycoplasma and chlamydia. A cytomegalovirus (CMV) titre was 16 on the first investigation at 5 months of age, but negative a few weeks later, indicating no CMV infection. Herpes simplex virus was maternally transferred, but the patient was not infected. Liver function tests were slightly elevated for aspartate aminotransferase (ASAT) and serum calcium, but were normal on follow-up a few weeks later and, hence, this was considered within the normal range.

\section{Task 2}

What would be your next investigation? Would you use any of the following:

1. HRCT scan of the chest.

2. Bronchoscopy.

3. Lung biopsy. 


\section{Answer 1}

An HRCT scan was performed.
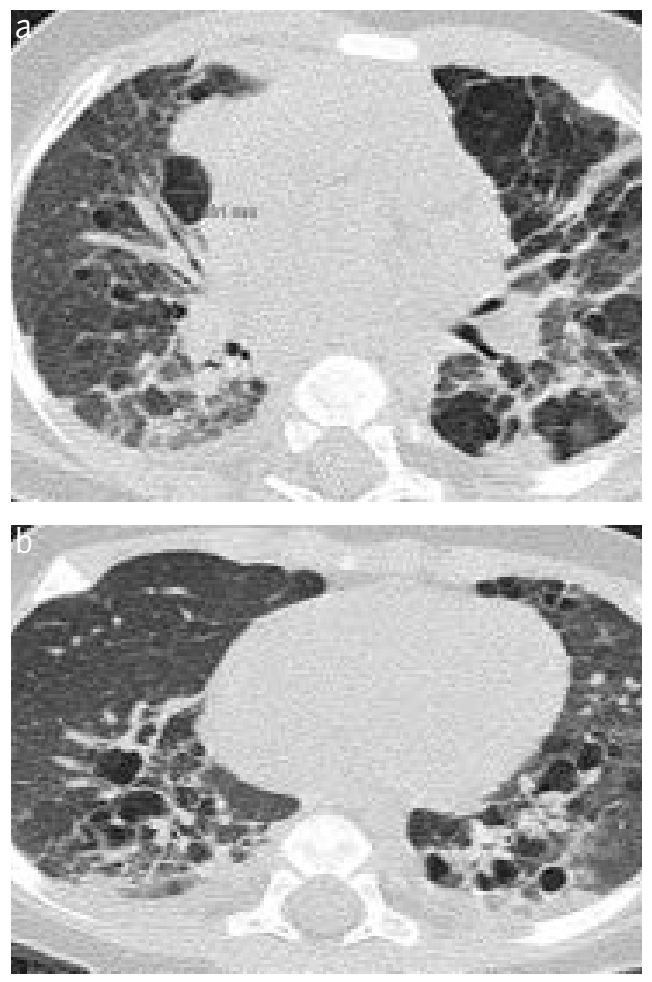

Figure 3

An HRCT scan of the patient.

The HRCT showed a bizarre picture. There were many "cystic" lesions, some up to almost 9 $\mathrm{mm}$, and diffused infiltrates widely distributed throughout the parenchyma. The only region that was actually spared was the costofrenic angle.

\section{Task 3}

\section{Considering the given information,} what is the most likely diagnosis?

\section{Cystic fibrosis.}

2. Langerhans cell histiocytosis

(LCH).

3. Interstitial pneumonitis.

4. Chronic lung disease of the newborn.

\section{Answer 2}

There was no evidence to support a diagnosis of chronic disease of the newborn. Interstitial pneumonitis is not usually associated with cystic lesions at such an early stage, so this was considered unlikely. An entirely negative sweat test made cystic fibrosis less likely as the primary diagnosis. Therefore, from the distribution and the sparing of the costofrenic angles [2], LCH was considered the most likely diagnosis.

\section{Likely diagnosis of LCH}

Primary or isolated LCH is uncommon, with few published reports in childhood [3-7]. Thus, we sought advice from the paediatric oncologist with two questions: first, how likely is this to be isolated pulmonary LCH in a 4-month-old infant and, secondly, what would be the recommended treatment? The responses to the first question from the international LCH Society varied from: "it's absolutely impossible" to "unlikely" or "it could most certainly be".

The treatment option suggested was chemotherapy in combination with systemic steroids.

Another issue was the possible link to the mother's Hodgkin's disease. There has been some speculation in the literature concerning a link between $\mathrm{LCH}$ and later Hodgkin's disease $[8,9]$ and the co-occurrence of $\mathrm{LCH}$ and Hodgkin's disease [10]; however, no report of a mother with Hodgkin's disease and LCH in her child has been found.

LCH is frequently a multi-organ or multi-system disease $[5,6,11]$, often involving cystic lesions of the skull. Thus, complete skeletal radiography was performed without evidence of cystic lesions elsewhere. 


\section{Further diagnostic procedures}

Although the diagnosis of LCH was believed to be highly likely at this stage, further diagnostic confirmation was required. A central venous catheter (CVK) was inserted during the general anaesthesia, for easy treatment access. A bone marrow aspiration showed some possible eosinophilic infiltrations, a finding that was not confirmed by a second opinion. No confirmative evidence was found from bone marrow examination, nor did bronchoalveolar lavage add further information.

\section{Open lung biopsy}

At age 5 months, an open lung biopsy (figure 4) was performed in the left lingular region that had been identified as pathological.

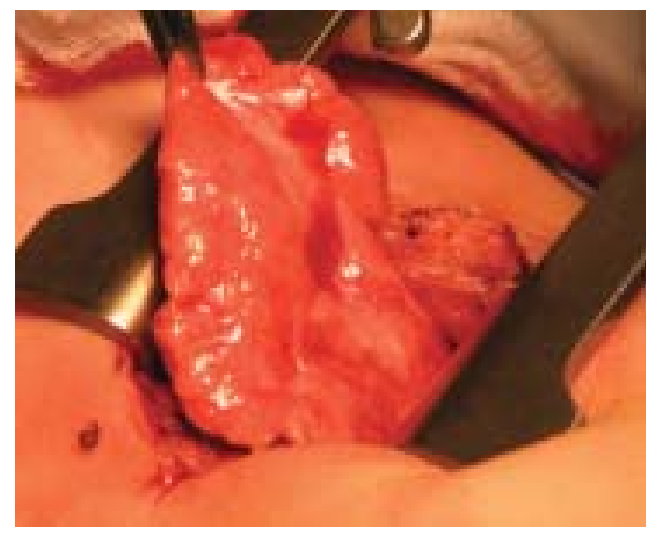

Figure 4

An open lung biopsy.

The biopsy demonstrated some lymphoid infiltration and the diagnosis was polyclonal lymphoid infiltration in the alveolar septa. In some of the alveolar septa, there were nodular lymphoid aggregates with focal germinal centres, and a few alveoli also had some macrophages and lipid macrophages. However, there was a relative sparing of the bronchioles and the terminal bronchioles, and no eosinophilia was found.

The follicular bronchiolitis that was observed was assessed also by pathologists elsewhere who suggested that the cystic lesions could be due to air trapping because of the infiltrations around the bronchioles. However, no evidence of CD1apositive cells was found for diagnosing $\mathrm{LCH}$ [2], and staining of the lung biopsy did not identify any signs of EBV, human herpes virus-8 or tuberculosis.

Therefore, the diagnosis was lymphocytic interstitial pneumonia (LIP), a diagnosis confirmed elsewhere.
However, the diagnosis from the open lung biopsy was not consistent with the bizarre CT scan. Furthermore, LIP is usually associated with immunodeficiency [12-15], which had not been demonstrated in the patient. Rather, there was an increase in CD3, CD4 and CD19 fractions of the cells and also of the CD8, i.e. an increase in most of the cell lines. T-cell function tests appeared to be normal in the CD3, CD4 and CD8 subset of cells.

For these reasons, the biopsy was re-evaluated to establish if this could actually be a start of Hodgkin's disease or other malignancy, but no monoclonal element was found, although there was a slight prominent band on the background of the polyclonal B-cells.

\section{Follow-up}

Clinically, about 6 weeks after referral (age 5 months), the patient was doing well, although he develop some cough, airways secretions and, once, had a very mild bronchial obstruction, which was successfully treated with inhaled racemic adrenaline. His lung function was unchanged, and oxygen saturation was still normal. The CVK was removed, since no treatment was started. However, the spleen had become palpable just below the left costal border, but ultrasound described a normally sized spleen at 6 months of age.

Therefore, at this stage, the patient had a diagnosis of LIP, he was gaining weight, was happy and developing well.

Task 4

What would you do now?

1. Observe and treat only if ill (the common recommendation). 2. Start treatment with pulse steroids and anti-fibrotic agents (the treatment for LIP). 3. Start treatment with inhaled steroids.

4. Wait and investigate further. 


\section{Answer 4 \\ This was a difficult decision. In view of the diag- nosis, the suggested treatment was high-dose i.v. pulse steroid therapy (methylprednisolone) and hydroxochloroquine. However, before starting such treatment, the diagnosis needs to be conclusive and the treatment should be ini- tiated at the appropriate time (diagnostic and clinical evaluation). \\ To be certain of the appropriate treatment, pos- sible progression of the disease was reaffirmed. A CT scan taken almost 2 months after initial scan was virtually unchanged, with bizarre find- ings. \\ The decision was therefore to observe the patient, and do an immediate CT scan and con- sider treatment if clinical deterioration was suspected. He had, in fact, developed some res- piratory symptoms with increased secretions and daily cough. \\ In the absence of well-documented treatment options, and in view of severe side-effects of sys- temic steroids or hydroxochloroquine, an experimental treatment with inhaled nebu- lised steroids (budesonide 1,000 $\mu$ g, four times per day) was commenced. The rationale was to try and achieve an anti-inflammatory effect without the potentially severe side-effects by systemic treatment.}

At 6 months the patient had developed a clinically palpable hepatosplenomegaly, confirmed by ultrasound.

\section{Task 5}

In view of the disease progress, what would be your next step?

1. Analyse urine for metabolic disease.

2. Perform a liver biopsy (the child's 4th general anaesthetic).

3. Perform a bone marrow biopsy.

4. All of the above.

5. Do an opthalmoscopy.

6. None, start treatment. 


\section{Answer 5 \\ Further investigations were performed. Urine for metabolic disease was normal. \\ An MR scan performed prior to the liver biopsy ascertained no further organ involvement. The bone marrow biopsy showed clear eosinophilic infiltrations and megakaryocyte hypoplasia.}

Thus, a metabolic disease could not be ruled out. A search of "Pub-med" for literature using "storage disease, lung, child" revealed 17 publications. However, since most of the glycogen storage diseases are associated with neurological impairment, this was not a likely diagnosis in the well-developing child. Lipid storage disease, type I Gaucher is associated with normal neurological development, but there was no evidence to support this diagnosis.

\section{Current development}

At age 15 months the child is doing very well. Respiratory rate is now within normal range, and he has had no respiratory symptoms for the last 3 months. The spleen is no longer palpably enlarged, whereas the liver is only slightly depressed. A CT scan performed at 13 months showed some improvement, especially with decreasing size of the "bullae". His weight and length gain is normal (follows his percentile from birth), his mental and physical development appears normal. The treatment presently is nebulised budesonide 1,000 mg twice daily.

Despite no absolute diagnosis, his physical development is excellent, and his prognosis is, therefore, optimistic.

\section{Acknowledgements}

We are indebted for help and advice in the management of this young infant to:

Jan Ericsson and Einar Hopp (radiologists), Ullevål University Hospital and the National Hospital of Norway, respectively. Marit Hellebostad (paediatric oncologist), Ullevål University Hospital. Erik Strøm and Borghild Roald (pathologists), the National Hospital of Norway and Ullevål University Hospital, respectively. Liv Osnes (immunologist), Ullevål University Hospital.

Kai-Håkon Carlsen (paediatrician), Voksentoppen, BKL, National Hospital of Norway. Andrew Bush and colleagues at the Brompton Hospital, London. Leland Fan and colleagues at the Texas Children's Hospital, Houston.

To see further discussion and questions concerning these findings go to the Breathe website at www.breathe-cme.org or email breathe@ersj.org.uk

\section{References}

1. Fauroux B, Epaud R, Clement A. Clinical presentation of interstitial lung disease in children. Paediatr Respir Rev 2004; 5: 98-100.

2. Sundar KM, Gosselin MV, Chung HL, Cahill BC. Pulmonary Langerhans cell histiocytosis: emerging concepts in pathobiology, radiology, and clinical evolution of disease. Chest 2003; 123: 1673-1683.

3. Chatkin JM, Bastos JC, Stein RT, Gaiger AM. Sole pulmonary involvement by Langerhans' cell histiocytosis in a child. Eur Respir J 1993; 6: 1226-1228.

4. McDowell HP, Macfarlane PI, Martin J. Isolated pulmonary histiocytosis. Arch Dis Child 1988; 63: 423-426.

5. Braier J, Latella A, Balancini B, et al. Outcome in children with pulmonary Langerhans cell histiocytosis. Pediatr Blood Cancer 2004; 43: 765-769.

6. Smets A, Mortele $K$, de Praeter G, Francois O, Benoit $Y$, Kunnen M. Pulmonary and mediastinal lesions in children with Langerhans cell histiocytosis. Pediatr Radiol 1997; 27: 873-876.

7. Schulze J, Kitz R, Gruttner HP, Schmidt H, Zielen S. Severe isolated pulmonary Langerhans cell histiocytosis in a 6-yearold girl. Eur J Pediatr 2004; 163: 320-322.

8. Sajjad SM, Luna MA. Primary pulmonary histiocytosis $X$ in two patients with Hodgkin's disease. Thorax 1982; 37: 110-113.

9. Shin MS, Buchalter SE, Ho KJ. Langerhans' cell histiocytosis associated with Hodgkin's disease: a case report. J Natl Med Assoc 1994; 86: 65-69.

10. Karadeniz C, Sarialioglu F, Gogus $S$, et al. Multiple primary malignancy: a report on Langerhans cell histiocytosis associated with Hodgkin's disease. Turk J Pediatr 1991; 33: 185-190.

11. Zeller B, Storm-Mathisen I, Smevik B, Lie SO. Multisystem Langerhans-cell histiocytosis with life-threatening pulmonary involvement - good response to cyclosporine A. Med Pediatr Oncol 2000; 35: 438-442.

12. Pitt J. Lymphocytic interstitial pneumonia. Pediatr Clin North Am 1991; 38: 89-95.

13. Teirstein AS, Rosen MJ. Lymphocytic interstitial pneumonia. Clin Chest Med 1988; 9: 467-471.

14. Koh DM, Hansell DM. Computed tomography of diffuse interstitial lung disease in children. Clin Radiol 2000; 55: 659-667.

15. Fishback N, Koss M. Update on lymphoid interstitial pneumonitis. Curr Opin Pulm Med 1996; 2: 429-433. 